\title{
How will telomeric complex be further contributed to our longevity? - The potential novel biomarkers of telomere complex counteracting both aging and cancer
}

\author{
Yiming $\mathrm{Lu}^{1^{*}}$, Bohua Wei ${ }^{1^{*}}$, Tao Zhang ${ }^{2}$, Zi Chen $^{3 凶}$, Jing Ye ${ }^{1,4 \bowtie}$ \\ ${ }^{1}$ Shanghai Ruijin Hospital, Shanghai Jiaotong University School of Medicine, Shanghai 200025, China \\ 2 Department of Laboratory Medicine, Huashan Hospital, Fudan University, Shanghai 200040, China \\ ${ }^{3}$ Department of Hematology, Huashan Hospital, Fudan University, Shanghai 200040, China \\ ${ }^{4}$ University of Nice, Laboratory of Biology and Pathology of Genomes, UMR 6267, CNRS U998, INSERM 28 avenue, \\ Valombrose Faculté de Médecine 06107, Nice Cedex 2, France \\ $\triangle$ Correspondence: drchenzi@tom.com (Z. Chen), yj11254@rjh.com.cn (J. Ye) \\ Received January 10, 2013 Accepted March 27, 2013
}

\begin{abstract}
With the smooth move towards the coming expected clinical reports of anticancer pharmaceutical molecules targeting telomeres and telomerase, and also with the exciting success in the extension of lifespan by regulating telomerase activity without increased onset of oncogenesis in laboratory mouse models (Garcia-Cao et al., 2006; Jaskelioff et al., 2011), we are convinced that targeting telomeres based on telomerase will be a potential approach to conquer both aging and cancer and the idea of longevity seems to be no more mysterious. More interestingly, emerging evidences from clinical research reveal that other telomeric factors, like specific telomeric binding proteins and nonspecific telomere associated proteins also show crucial importance in aging and oncogenesis. This stems from their roles in the stability of telomere structure and in the inhibition of DNA damage response at telomeres. Uncapping these proteins from chromosome ends leads to dramatic telomere loss and telomere dysfunction which is more abrupt than those induced by telomerase inactivation. Abnormal expression of these factors results in developmental failure, aging and even oncogenesis evidenced by several experimental models and clinical cases, indicating telomere specific proteins and its associated proteins have complimentary roles to telomerase in telomere protection and controlling cellu-
\end{abstract}

lar fate. Thus, these telomeric factors might be potential clinical biomarkers for early detection or even therapeutic targets of aging and cancer. Future studies to elucidate how these proteins function in telomere protection might benefit patients suffering aging or cancer who are not sensitive to telomerase mediation.

KEYWORDS telomere, telomerase, oncogenesis, senescence, telomere binding protein

\section{INTRODUCTION}

Owing to the contribution of telomere and telomerase study in the medical development of longevity, the scientific pioneers who discovered telomerase have been awarded the Nobel Prize in 2009. The beginning of telomere study can be dated back to 1960s, and the research on telomeres and telomerase since the past decades reveals why we have a limited life and shed light on how we can improve our life span (Bernardes de Jesus and Blasco, 2000; Jaskelioff et al., 2011; Gunes and Rudolph, 2013) (Fig. 1).

\section{HOW TO EXPAND LIFE SPAN BY TARGETING TELOMERASE?}

In eukaryotic cells, telomeres are genetic code of repeated TTAGGG sequences at lineal chromosome ends to protect genome integrity. Telomere function is regulated by telomere

\footnotetext{
*These authors contributed equally to the work.
} 


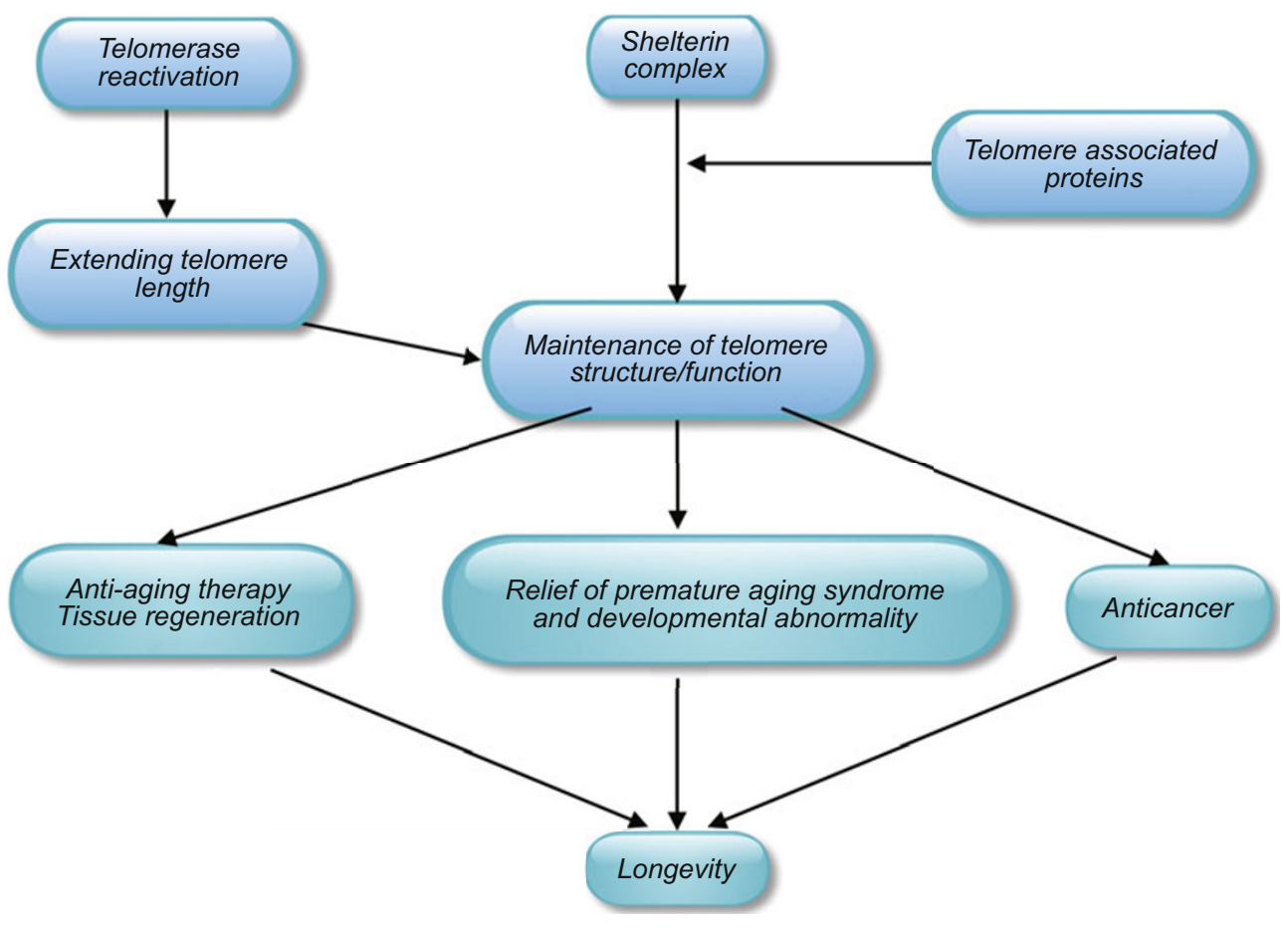

Figure 1. The role of telomeric nucleocomplex in longevity.

length and telomere structure. Due to the replicative problems, telomeres shorten during each cell division. After 40-80 doubling populations, telomeres reach a critical length, and telomere dysfunction exposes the natural chromosome ends to the DNA damage response (DDR) machinery. The activation of DDR recruits $\mathrm{YH} 2 \mathrm{AX}$ and 53BP1, the key transducers of the DNA damage checkpoint to form foci at telomeres, which is called telomere dysfunction foci (TIF) (Gilson and SegalBendirdjian, 2010; Ye et al., 2010b). They activate downstream checkpoint kinases to induce growth arrest, apoptosis and replicative senescence (d'Adda di Fagagna et al., 2003). Thus, the protective function of telomeres is limited by the number of cellular passage and the initial telomere length is a biomedical utensil to predict the individual's nature longevity (Fig. 2).

Accumulation of short telomeres and dysfunctional telomeres due to the increased age result in stem cell dysfunction and tissue degeneration due to the replicative senescence in cells with normal checkpoint activation system (Rudolph et al., 1999). In checkpoint deficient cells, critical short telomeres induced telomere dysfunction results in tumorigensis (Chin et al., 1999; Artandi et al., 2000). Hence, telomeres transmit the crosstalk between aging and cancer, and this could explain why most types of cancers are regarded as aging associated diseases to a certain extent.

Telomerase, a ribonuclease enzyme, contains a reverse transcriptase (hTERT), which catalyzes its own RNA component as template to elongate short telomeres at a certain length (Greider and Blackburn, 1985). Like telomere length, telomerase activity is also crucial for both tissue regeneration and malignant infiltration.

Telomerase activity is indispensible for the reproduction of stem cell, germ line cells and high proliferative cell (Lee et al., 1998). Mouse models owing to the telomerase RNA deficiency result in premature aging (Blasco et al., 1997). These $\mathrm{TR}^{-/-}$mice showed wide range of degenerative pathologies and decreased longevity in their later generation due to critical short telomeres and chromosome instability. These degenerative diseases include aplastic anemia, lung fibrosis, xeroderma pigmentosum, intestinal atrophy, germ line depletion and high incidence of oncogenesis, etc. (Rudolph et al., 1999). Clinical study also identified reduced hTERT level or mutated hTERT in patients with dyskeratosis congenita (DKC) (Shay and Wright, 2004). All these indicate that telomerase induced telomere elongation is an essential step for tissue development and renewal capacity and telomerase reactivation is supposed to be a possible strategy to reverse physiological aging. Indeed, exogenous replenishment of telomerase is a possible therapeutic strategy to counteract telomere associated degenerative disease and reverse physiological aging (Garcia-Cao et al., 2006; Jaskelioff et al., 2011).

Telomerase is crucial for tumor progression. Experiments have shown that over-expression of exogenous hTERT in normal primary fibroblast cells results in immortalization. Telomerase reactivation in transformed cells accelerates malignant progression and infiltration. The proliferation of malignant transformed cells due to critical short telomeres can be repressed in telomerase negative cells, but accelerated when telomerase is reactivated (Bodnar et al., 1998; Seger et al., 2002). Indeed, 


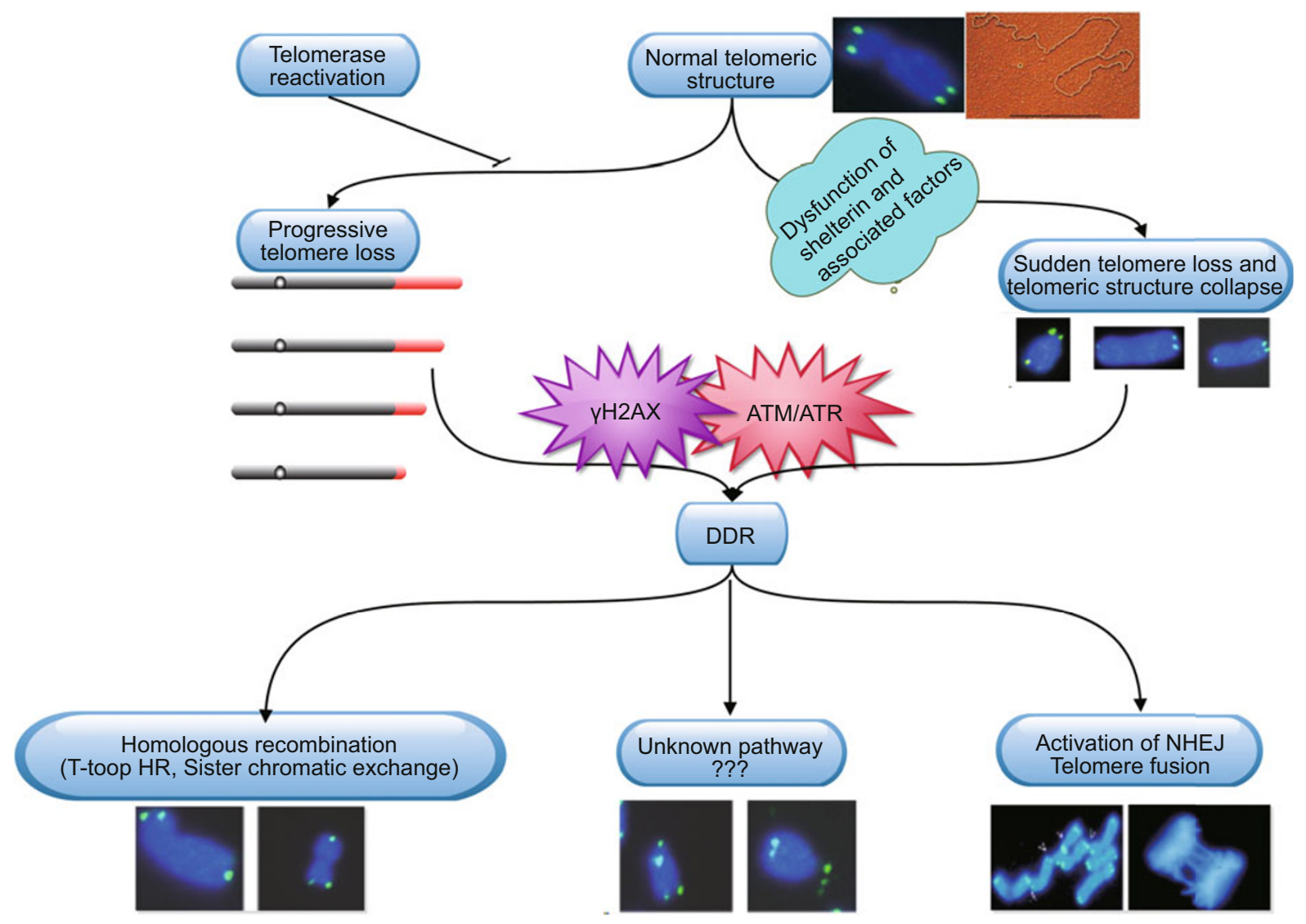

Figure 2. Causes and consequences of telomere dysfunction.

telomerase is reactivated in $85 \%-95 \%$ of tumor cells and clinical advanced cancer tissues, but it is undetectable in normal somatic cells (Kim et al., 1994; Wright et al., 1996). hTERT promoter mutation further prohibit telomere function and is responsible for certain cancer mobidity (Horn et al., 2013; Huang et al., 2013). Telomeric repeat amplification protocol (TRAP) assay is a sensitive and specific method to detect telomerase activity in cultured cells and clinical tissue samples, which facilitates the translation of telomerase study from lab to bedside (Wright et al., 1995). Telomerase activity is supposed to be a hopeful medical biomarker for the surveillance of advanced tumor.

In summary, telomere loss is rate limiting for nature longevity. Telomere shortening induced by telomerase inactivation is implicated in the replicative senescence induced renewal inability of stem cells or regeneration failure of high proliferative organs, and telomerase reactivation in telomere dysfunction cells facilitates malignant proliferation. Thus, biomedical research targeting telomerase could be a double-edged sword for both anti-senescent therapy and anticancer therapy. Indeed, the fact that pathological aging in telomerase deficient mice can be reversed with telomerase reactivation, strongly encourages the further study on telomerase based drug development (Garcia-Cao et al., 2006; Jaskelioff et al., 2011). This will not only give hope to the treatment of congenital presaging syndrome and aging associated diseases, but also support the idea that extending health-span might be no more a fancy. The novel anticancer drugs targeting telomerase with different pharmaceutical principles have already entered clinical study. For example, the small molecule hTERT inhibitor BIBR1532 and GRN163L, a thio-phosphoramidate oligonucleotide targeting the template region of hTR as a "template antagonist" and GV1001, a telomerase-specific, promiscuous class II peptide vaccine are currently in an advanced stage of clinical development (Shaw et al., 2010; Schlapbach et al., 2011). More importantly, mouse models with the combination of overexpression of TERT and tumor suppressor genes (Sp53/Sp16SARF/TgTERT) is supposed to delay aging, extend mice longevity and be resistant to oncogenesis, indicating mediation of telomerase is a key point for genotherapy of longevity (Garcia-Cao et al., 2006; Jaskelioff et al., 2011).

\section{TELOMERE SPECIFIC BINDING PROTEINS EMERGE AS NOVEL HALL MARKERS IN AGING RELATED DISEASES AND ARE POTENTIAL CANDIDATES FOR CLINICAL TREATMENT IN TELOMERE DYSFUNCTION INDUCED DISEASES}

Besides the gradual telomere loss in systematic organismal 


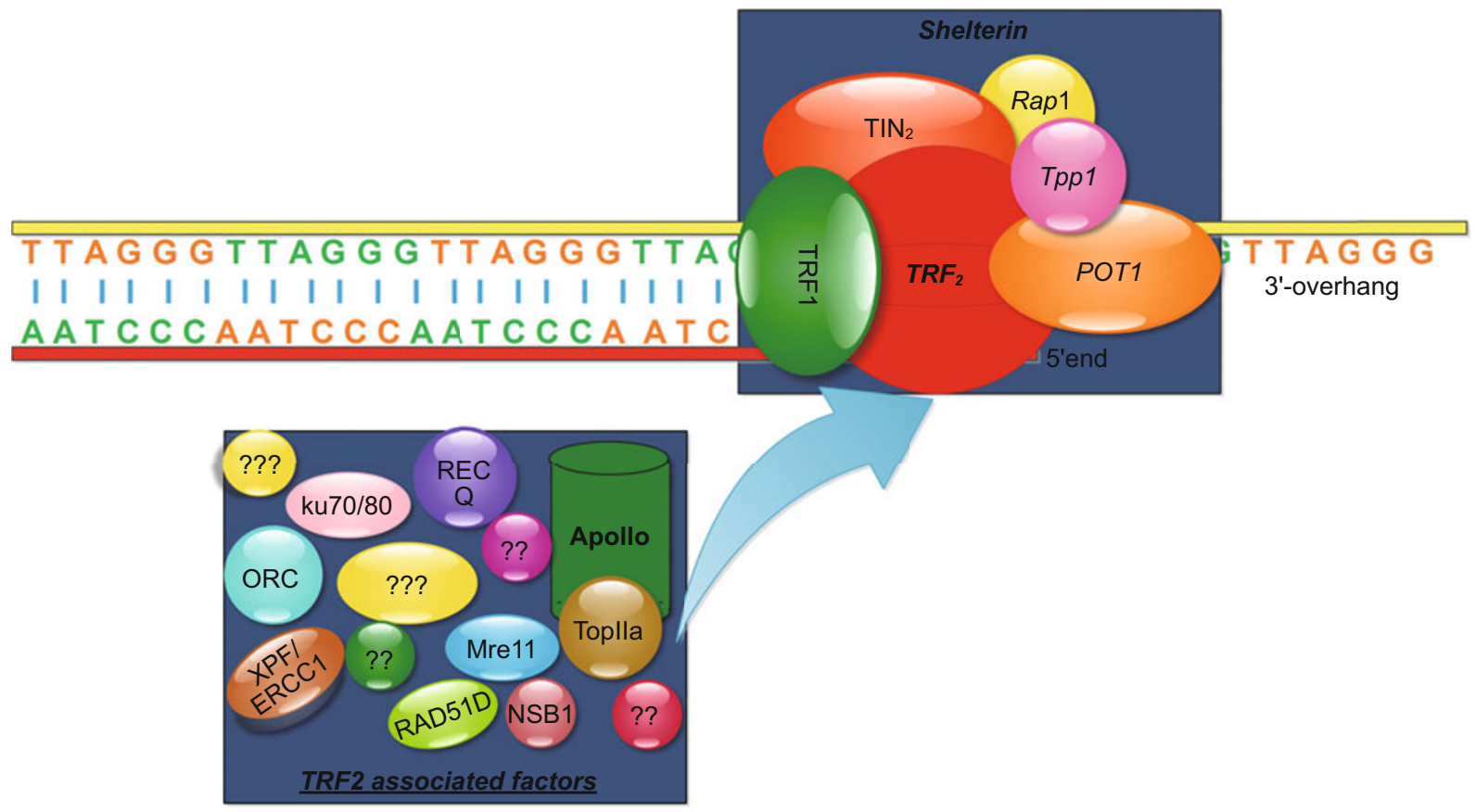

Figure 3. Structure of telomeres and representative telomeric associated factors.

aging, telomere sequence has been found lost dramatically independent of replicative telomere dynamics (Karlseder et al., 2002). This is due to the dysfunction of telomere binding proteins, which bind specific to chromosome ends to cap the telomeric fragile structure (van Steensel et al., 1998). These telomere capping proteins include TRF1, TRF2, POT1, TIN2, TPP1 and RAP1. They form a protein complex and also known as shelterin/telosome (de Lange, 2005; Xin et al., 2008) (Fig. 3).

Shelterin is involved in the maintenance of telomere length and telomere structure. TRF1 and TRF2 bind to the double strand of telomeres, whereas POT1 binds to the single strand of telomeres. They negatively regulate telomere length (Palm and de Lange, 2008). TRF2 is involved in the organization of t-loop, a duplex lariat structure through strand invasion of 3 '-overhang to avoid telomere erosion (Griffith et al., 1999; Stansel et al., 2001; Wang et al., 2004). POT1 regulates telomerase access to telomeres with TPP1 (Loayza and De Lange, 2003; Wang et al., 2007; Nandakumar et al., 2012). TRF1 is also involved in the telomeric repeat-containing RNA transcription (TERRA) and recent results have shown that TRF1 as well as TRF2 are crucial for telomere maintenance during replicative stress (Schoeftner and Blasco, 2008; Martinez et al., 2009; Munoz et al., 2009). Knockout shelterin components in mouse embryonic fibroblast (MEF) or inhibition of the expression of these proteins or overexpression of dominant negative alleles of these proteins in vitro or in cultured human cell lines lead to the collapse of telomere structures, including sudden telomere loss, t-loop recombination and telomere fusion (Karlseder et al., 1999; Wang et al., 2004).

One of the essential functions of shelterin is to cap telom- eres from being recognized by DNA damage response. They play an important role in the inhibition of ATM or ATR activation at telomeres, which can phosphate the downstream Chk1 and Chk2 and finally block cell cycle division (Karlseder et al., 1999, 2004; Denchi and de Lange, 2007). Shelterin also represses DNA repair machinery, including non-homologous ending joining (NHEJ), homologous recombination (HR) and other repair pathway. These repair machineries in TRF2 compromised cells result in chromosome end fusion, telomere sister chromatid exchange and t-loop HR which threaten telomere integrity (van Steensel et al., 1998; Karlseder et al., 1999; Smogorzewska et al., 2002) (Fig. 2).

In vivo evidences also demonstrate that shelterin proteins are physiological elements essential for the mammalian development and tissue function. TRF2 knockout mice are embryonic lethal. TRF1, TPP1 and RAP1 conditional knockout mice show presaging syndrome and high incidence of oncogenesis (Martinez et al., 2009, 2010). POT1 deletion induces mouse DKC (Hockemeyer et al., 2008). These knockout mice display abnormal syndrome even in normal telomere length, which indicates that individuals with telomere dysfunction induced by the depletion of shelterin components might exhibit more severe and rapid pathological aging phenotypes than those induced by telomerase deficiency. In consistence with mouse models, genetic variation in shelterin components has been observed in patients with cancer and pathological aging. One SNP of TRF1 gene is significantly associated with melanoma, and mutated TRF1 allele has been observed in aplastic anemia. TIN2 mutations have been found in DKC patients (Walne et al., 2008; Sasa et al., 2012). There is no report on patho- 
logical SNP in TRF2 gene which might be due to the fact that TRF2 mutation is embryonic lethality. This suggests that TRF2 might play a center role in shelterin and the absence of TRF2 might cause a more vulnerable tissue development or regeneration failure compared to other shelterin components. All these indicate that shelterin is crucial for telomere function and the abolishment of these proteins results in genomic instability as severe as telomere loss due to cell passage or due to the absence of telomerase activity. It might have more exacerbated consequences, because it occurs more brutally than progressive telomere loss. TRF2, together with other shelterin components, are potential biomarkers for aging pathologies.

Although shelterin components play a complimentary role to telomerase through capping telomere structure and stabilizing telomeres, higher dosage of shelterin components act opposite to its protective function. Overexpression of TRF2 or TRF1 in $\mathrm{K} 5$ mice lead to telomere loss and chromosome aberration (Munoz et al., 2009), presaging syndrome and tumorgenesis. This is concomitant with clinical reports that TRF2 and TRF1 levels are abnormally increased in pathological samples of gastric cancer, skin cancer, lung cancer, etc. (Munoz et al., 2005; Ning et al., 2006; Hsu et al., 2007). In addition, telomerase deficiency in K5-TRF2 mice accelerates TRF2 induced carcinogenesis, strongly supports that TRF2 overexpression could spontaneously generate a fully transformed tumorigenic phenotype, whereas telomerase activity is dispensible during malignant transformation (Blanco et al., 2007). Thus, TRF2 overexpression acts as a potent oncogenic event. Targeting shelterin components, especially TRF2 might be a novel anticancer strategy.

\section{Telomere associated proteins are taking the lead in telomere protection}

Shelterin members perform their role in telomere protection through interacting with a large number of associated proteins. These shelterin accessory factors are mainly involved in nontelomeric functions, including DNA damage signal (Mre11 complex, 911 complex, etc.), DNA repair (ku70/80, XPF/ERCC1, Apollo, RAD51D, etc.) and DNA replication (ORC, RecQ, Toplla, etc.). They are recruited to induce their function at telomeres by the special modification of shelterin (Fig. 3).

Recently, emerging evidences suggest that one of these associated proteins, SNM1B/APOLLO interacts with TRF2 and plays an important role in telomere protection (Lenain et al., 2006; van Overbeek and de Lange, 2006; Ye et al., 2010a). SNM1B/APOLLO is a $5^{\prime}$-exonuclease belonging to the metallo$\beta$-lactamase family. Besides Apollo, the metallo- $\beta$-lactamase family, which has a common domain of $\beta$-CPSF-ArtemisSnm1-Pso2, contains SNM1A and SNM1C/Artemis, and all of them are involved in DDR and repair system via their nuclease activity. SNM1A mediates radiation-induced DNA repair. SN$\mathrm{M} 1 \mathrm{C} / \mathrm{Artemis}$ localizes and degrades the hairpins generated during $\mathrm{V}(\mathrm{D}) \mathrm{J}$ recombination. SNM1C/Artemis is also responsible for the NHEJ repair and IR-induced DNA damage. SNM1B/
APOLLO is involved in ICL repair.

Five main papers publishing at nearly the same time tried to elucidate how Apollo protects telomeres, in addition to its role in ICL repair. Apollo dysfunction induced either by RNA interference or by overexpression of nuclease dead alleles leads to robust increase in TIF and various types of telomere aberration, including telomere free ends, chromatid fusion especially leading hand chromatid fusion, and fragile telomeres. Recent results have shown that the 5'-exonuclease activity of Apollo has been demonstrated crucial for telomere processing during replication. It might be involved in the progression of replication fork to maintain the inner part of telomeres through removal of the incorrect replicative products. Thus, Apollo might be implicated in the prevention of fork collapse or retrogression at telomeres. This 5 '-exonuclease activity is also shown to relieve the positive supercoil structure ahead of fork induced by topoisomerase II depletion (Ye et al., 2010a). Besides its role in telomere replication, Apollo is also crucial for telomere processing after replication. Apollo knockout in mouse embryonic fibroblast cells shows 3 '-overhang loss and telomere fusion in leading strand, which is regulated by TPP1, POT1 and other associated factors (Lam et al., 2010; Wu et al., 2010).

Several results show that Apollo protects telomeres through repressing ATM, whereas others didn't elicit the direct link that Apollo modulates ATM activation. These inconsistent results might be due to the variant cell models they used in experiment assay, for example, human cancer cell lines or mouse embryonic cells or due to the inhibiting efficiency of the nucleases activity of Apollo (Demuth et al., 2008; Lam et al., 2010; Wu et al., 2012). Thus, it is still elusive whether Apollo is responsible for the inhibition of ATM or other DDR pathway induced at telomeres.

Apollo is also involved in the inhibition of NHEJ at uncapped telomeres. Apollo dysfunction in TRF2 compromised cells results in exacerbation of chromosome end fusion induced by NHEJ (Ye et al., 2010a). The high levels of telomeric endto-end fusions owing to Snm1B/Apollo mutation observed in mouse embryonic fibroblasts (MEFs) impairs cell proliferation and results in cell senescence or apoptosis (van Overbeek and de Lange, 2006; Lam et al., 2010).

Recently, the above function of Snm1B/Apollo has been demonstrated in vivo experimental study and clinical reports. Snm1B/Apollo homozygous null mice die at birth with developmental delay and defects in multiple organ systems in p53 independent pathway (Akhter et al., 2010). Moreover, a clinical case also shows that a unique Apollo splice variant was identified in a severe Hoyeraal-Hreidarsson $(\mathrm{HH})$ syndrome patient who is characterized severe premature aging, bone marrow failure, and immunodeficiency (Touzot et al., 2010), indicating such a transcriptional variant of Apollo does result in pathological telomere dysfunction. Moreover, a mutated DCLRE1B gene of Apollo identified with a breakpoint within the intron 3 of DCLRE1B in the Wilms tumors, a nephroblastoma that typically affects children (Natrajan et al., 2007). These two clinical studies attract the attention of telomere discoveries, and high- 


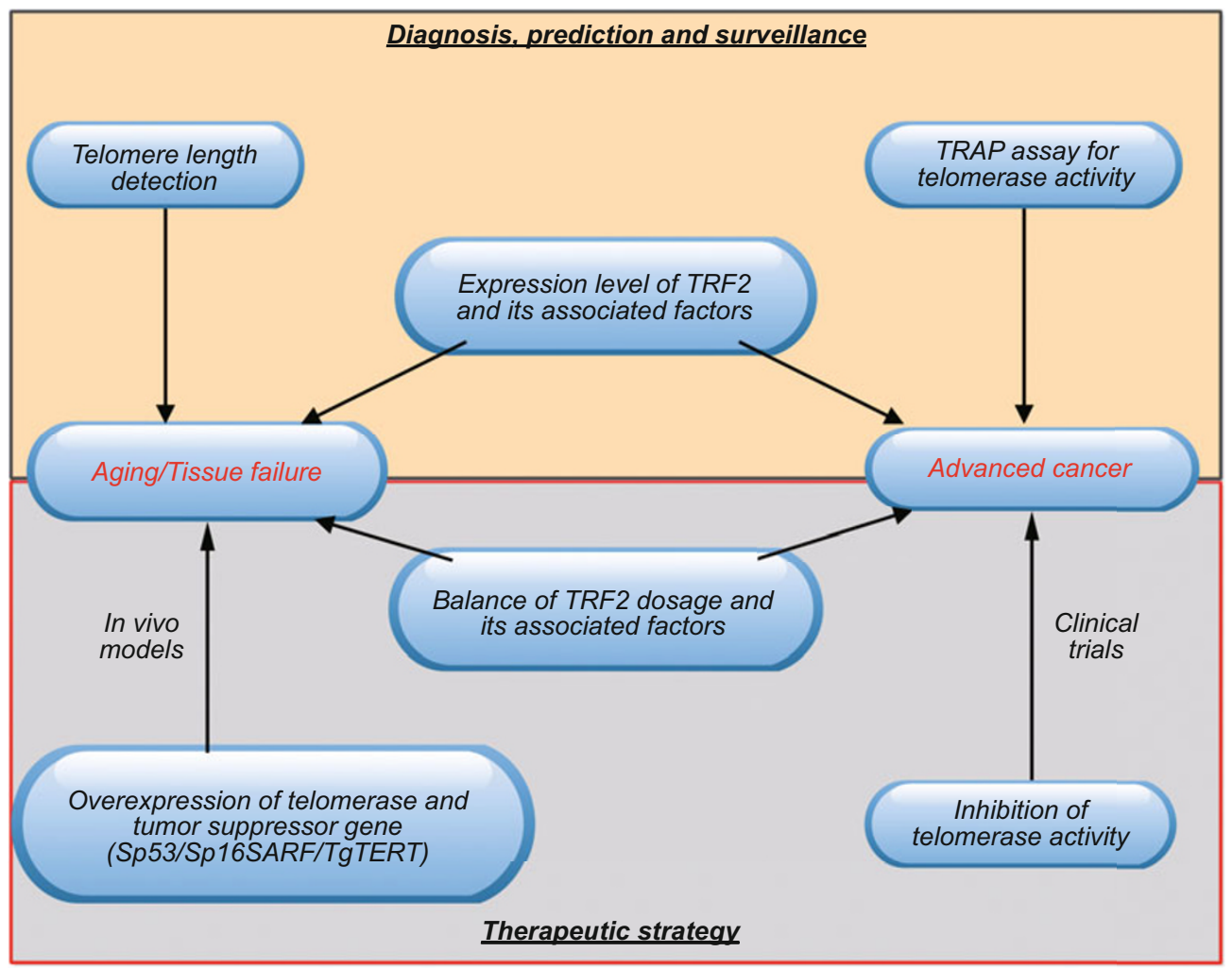

Figure 4. The potential clinical value of telomeres in longevity.

light that Apollo is a crucial physiological protein in telomere physiology and tissue development. Apollo interacts with TRF2 and TPP1, maybe other shelterin members during various steps of telomere replication to maintain telomere integration and protect against DDR machinery and DNA repair machinery.

Overall, telomeric complex, including specific telomeric sequences and its nucleoprotein capping structure is crucial element responsible for life span by eliminating cancer and aging associated degenerative pathologies. Thus, targeting telomeres might be a hopeful strategy independent telomerase to counteract a wide range of diseases of variant tissue origin including cancer, stem cell dysfunction and tissue regenerative inability.

\section{CONCLUSION}

No biomarkers exist that has been associated with so broad spectrum of affected diseases and organs as telomere complex. Telomere length is regarded as the mitotic alarm for the renewal capacity of stem cell and tissue regeneration. Telomerase activity is even more sensitive than p53 mutation as a clinical biomarker of tumor infiltration. Also, targeting telomere length and telomerase could be an effective pharmaceutical strategy to cure aging related diseases, including tissue degeneration and cancer, regardless of tissue specificity and clinical manifestation. Thus, managing telomere length or the expression of telomerase controls the origin of these diseases. Nowadays, pharmaceutical molecules targeting telomerase for cancer therapy is ongoing for clinical trials and the strategy to extend telomere length by telomerase reactivation has been proven in the mouse model to improve tissue function and extend life span. The next crucial problem is to solve whether exogenous expression of telomerase in dysfunctional tissue should be balanced to avoid malignant proliferation besides its main function to gain re-acquired renewal capacity. Fortunately, the combined overexpression of telomerase and tumor suppression gene in mouse model has been demonstrated as a solution for longevity without increased onset of oncogenesis. Therefore, mediation of telomerase activity is not an extravagant hope to reach the final destination of longevity with health and life quality (Fig. 4).

More importantly, shelterin and their associated proteins have displayed their compliment role in aging and cancer to telomerase, evidenced by clinical samples and mouse models. Moreover, the ablation of these proteins result in more dramatic phenotype in telomere dysfunction and tissue degeneration, which exhibit more rapidly than those induced by telomerase deficiency. This indicates that targeting these proteins might be more sensitive and effective than or at least the same as targeting telomerase, especially for those who are antagonistic to telomerase intervention. Thus, it is highly hypothesized that 
these proteins could be potential clinical biomarkers of aging or cancer, and the study on these proteins might enable us benefit more from telomeres to discover new drugs for longevity (Fig. 4).

TRF2 and Apollo are just two examples, from which we know that shelterin and their associated proteins are crucial for telomere function, as well as aging and cancer. Other shelterin proteins and their associated proteins, like TIN2, TRF1 or WRN, BLM, etc. are also implicated in presaging syndrome and cancer, although the molecular mechanisms might be various. Thus, further studies are necessary to identify the function of these proteins in telomere protection. Although several clinical studies have demonstrated that the expression level of these proteins are associated with clinical aging and cancer, further clinical studies of large scale investigation in multicenter are needed to verify their expression level in patients. It is hopeful that shelterin and their associated proteins will provoke a new era of telomere associated biomedical study by discovering telomeric protein based diagnostic biomarker and therapeutic strategy.

\section{ACKNOWLEDGEMENTS}

This work was supported by the National Natural Science Foundation of China (Grant Nos. 81270433, 81170491, and 81000875), and the Foundation for Young Scientist in Shanghai Municipal Health Bureau in China (Grant No. 2010.24).

\section{ABBREVIATIONS}

DKC, dyskeratosis congenital; HH, Hoyeraal-Hreidarsson; HR, homologous recombination; MEF, mouse embryonic fibroblast; NHEJ, non-homologous ending joining; TIF, telomere dysfunction foci; TRAP, telomeric repeat amplification protocol

\section{COMPLIANCE WITH ETHICS GUIDELINES}

Yiming Lu, Bohua Wei, Tao Zhang, Zi Chen, and Jing Ye declare that they have no conflict of interest.

This article does not contain any studies with human or animal subjects performed by the any of the authors.

\section{REFERENCES}

Akhter, S., Lam, Y.C., Chang, S., and Legerski, R.J. (2010). The telomeric protein SNM1B/Apollo is required for normal cell proliferation and embryonic development. Aging Cell 9, 1047-1056.

Artandi, S.E., Chang, S., Lee, S.L., Alson, S., Gottlieb, G.J., Chin, L., and DePinho, R.A. (2000). Telomere dysfunction promotes nonreciprocal translocations and epithelial cancers in mice. Nature 406, 641-645.

Bernardes de Jesus, B., and Blasco, M.A. (2011). Aging by telomere loss can be reversed. Cell Stem Cell 8, 3-4.

Blanco, R., Munoz, P., Flores, J.M., Klatt, P., and Blasco, M.A. (2007). Telomerase abrogation dramatically accelerates TRF2-induced epithelial carcinogenesis. Genes Dev 21, 206-220.

Blasco, M.A., Lee, H.W., Hande, M.P., Samper, E., Lansdorp, P.M., DePinho, R.A., and Greider, C.W. (1997). Telomere shortening and tumor formation by mouse cells lacking telomerase RNA. Cell 91, 25-34.

Bodnar, A.G., Ouellette, M., Frolkis, M., Holt, S.E., Chiu, C.P., Morin, G.B., Harley, C.B., Shay, J.W., Lichtsteiner, S., and Wright, W.E. (1998). Extension of life-span by introduction of telomerase into normal human cells. Science 279, 349-352.

Chin, L., Artandi, S.E., Shen, Q., Tam, A., Lee, S.L., Gottlieb, G.J., Greider, C.W., and DePinho, R.A. (1999). p53 deficiency rescues the adverse effects of telomere loss and cooperates with telomere dysfunction to accelerate carcinogenesis. Cell 97, 527-538.

d'Adda di Fagagna, F., Reaper, P.M., Clay-Farrace, L., Fiegler, H., Carr, P., Von Zglinicki, T., Saretzki, G., Carter, N.P., and Jackson, S.P. (2003). A DNA damage checkpoint response in telomere-initiated senescence. Nature 426, 194-198.

de Lange, T. (2005). Shelterin: the protein complex that shapes and safeguards human telomeres. Genes Dev 19, 2100-2110.

Demuth, I., Bradshaw, P.S., Lindner, A., Anders, M., Heinrich, S., Kallenbach, J., Schmelz, K., Digweed, M., Meyn, M.S., and Concannon, P. (2008). Endogenous hSNM1B/Apollo interacts with TRF2 and stimulates ATM in response to ionizing radiation. DNA Repair (Amst) 7, 1192-1201.

Denchi, E.L., and de Lange, T. (2007). Protection of telomeres through independent control of ATM and ATR by TRF2 and POT1. Nature 448, 1068-1071.

Garcia-Cao, I., Garcia-Cao, M., Tomas-Loba, A., Martin-Caballero, J., Flores, J.M., Klatt, P., Blasco, M.A., and Serrano, M. (2006). Increased p53 activity does not accelerate telomere-driven ageing. EMBO Rep 7, 546-552.

Gilson, E., and Segal-Bendirdjian, E. (2010). The telomere story or the triumph of an open-minded research. Biochimie 92, 321-326.

Greider, C.W., and Blackburn, E.H. (1985). Identification of a specific telomere terminal transferase activity in Tetrahymena extracts. Cell 43, 405-413.

Griffith, J.D., Comeau, L., Rosenfield, S., Stansel, R.M., Bianchi, A., Moss, H., and de Lange, T. (1999). Mammalian telomeres end in a large duplex loop. Cell 97, 503-514.

Gunes, C., and Rudolph, K.L. (2013). The role of telomeres in stem cells and cancer. Cell 152, 390-393.

Hockemeyer, D., Palm, W., Wang, R.C., Couto, S.S., and de Lange, T. (2008). Engineered telomere degradation models dyskeratosis congenita. Genes Dev 22, 1773-1785.

Horn, S., Figl, A., Rachakonda, P.S., Fischer, C., Sucker, A., Gast, A., Kadel, S., Moll, I., Nagore, E., Hemminki, K., et al. (2013). TERT promoter mutations in familial and sporadic melanoma. Science 339, 959-961.

Hsu, C.P., Ko, J.L., Shai, S.E., and Lee, L.W. (2007). Modulation of telomere shelterin by TRF1 [corrected] and TRF2 interacts with telomerase to maintain the telomere length in non-small cell lung cancer. Lung Cancer 58, 310-316.

Huang, F.W., Hodis, E., Xu, M.J., Kryukov, G.V., Chin, L., and Garraway, L.A. (2013). Highly recurrent TERT promoter mutations in human melanoma. Science 339, 957-959.

Jaskelioff, M., Muller, F.L., Paik, J.H., Thomas, E., Jiang, S., Adams, A.C., Sahin, E., Kost-Alimova, M., Protopopov, A., Cadinanos, J., et al. (2011).Telomerase reactivation reverses tissue degeneration in aged telomerase-deficient mice. Nature 469, 102-106.

Karlseder, J., Broccoli, D., Dai, Y., Hardy, S., and de Lange, T. (1999). p53- and ATM-dependent apoptosis induced by telomeres lacking 
TRF2. Science 283, 1321-1325.

Karlseder, J., Hoke, K., Mirzoeva, O.K., Bakkenist, C., Kastan, M.B., Petrini, J.H., and de Lange, T. (2004). The telomeric protein TRF2 binds the ATM kinase and can inhibit the ATM-dependent DNA damage response. PLoS Biol 2, E240.

Karlseder, J., Smogorzewska, A., and de Lange, T. (2002). Senescence induced by altered telomere state, not telomere loss. Science 295, 2446-2449.

Kim, N.W., Piatyszek, M.A., Prowse, K.R., Harley, C.B., West, M.D., Ho, P.L., Coviello, G.M., Wright, W.E., Weinrich, S.L., and Shay, J.W. (1994). Specific association of human telomerase activity with immortal cells and cancer. Science 266, 2011-2015.

Lam, Y.C., Akhter, S., Gu, P., Ye, J., Poulet, A., Giraud-Panis, M.J., Bailey, S.M., Gilson, E., Legerski, R.J., and Chang, S. (2010). SNMIB/ Apollo protects leading-strand telomeres against NHEJ-mediated repair. EMBO J 29, 2230-2241.

Lee, H.W., Blasco, M.A., Gottlieb, G.J., Horner, J.W., 2nd, Greider, C.W., and DePinho, R.A. (1998). Essential role of mouse telomerase in highly proliferative organs. Nature 392, 569-574.

Lenain, C., Bauwens, S., Amiard, S., Brunori, M., Giraud-Panis, M.J., and Gilson, E. (2006). The Apollo 5' exonuclease functions together with TRF2 to protect telomeres from DNA repair. Curr Biol 16, 1303-1310.

Loayza, D., and De Lange, T. (2003). POT1 as a terminal transducer of TRF1 telomere length control. Nature 423, 1013-1018.

Martinez, P., Thanasoula, M., Carlos, A.R., Gomez-Lopez, G., Tejera, A.M., Schoeftner, S., Dominguez, O., Pisano, D.G., Tarsounas, M., and Blasco, M.A. (2010). Mammalian Rap1 controls telomere function and gene expression through binding to telomeric and extratelomeric sites. Nat Cell Biol 12, 768-780.

Martinez, P., Thanasoula, M., Munoz, P., Liao, C., Tejera, A., McNees, C., Flores, J.M., Fernandez-Capetillo, O., Tarsounas, M., and Blasco, M.A. (2009). Increased telomere fragility and fusions resulting from TRF1 deficiency lead to degenerative pathologies and increased cancer in mice. Genes Dev 23, 2060-2075.

Munoz, P., Blanco, R., de Carcer, G., Schoeftner, S., Benetti, R., Flores, J.M., Malumbres, M., and Blasco, M.A. (2009). TRF1 controls telomere length and mitotic fidelity in epithelial homeostasis. Mol Cell Biol 29, 1608-1625.

Munoz, P., Blanco, R., Flores, J.M., and Blasco, M.A. (2005). XPF nuclease-dependent telomere loss and increased DNA damage in mice overexpressing TRF2 result in premature aging and cancer. Nat Genet 37, 1063-1071.

Nandakumar, J., Bell, C.F., Weidenfeld, I., Zaug, A.J., Leinwand, L.A., and Cech, T.R. (2012). The TEL patch of telomere protein TPP1 mediates telomerase recruitment and processivity. Nature 492, 285-289.

Natrajan, R., Williams, R.D., Grigoriadis, A., Mackay, A., Fenwick, K., Ashworth, A., Dome, J.S., Grundy, P.E., Pritchard-Jones, K., and Jones, C. (2007). Delineation of a $1 \mathrm{Mb}$ breakpoint region at $1 \mathrm{p} 13$ in Wilms tumors by fine-tiling oligonucleotide array CGH. Genes Chromosomes Cancer 46, 607-615.

Ning, H., Li, T., Zhao, L., Li, T., Li, J., Liu, J., Liu, Z., and Fan, D. (2006). TRF2 promotes multidrug resistance in gastric cancer cells. Cancer Biol Ther 5, 950-956.

Palm, W., and de Lange, T. (2008). How shelterin protects mammalian telomeres. Annu Rev Genet 42, 301-334.
Rudolph, K.L., Chang, S., Lee, H.W., Blasco, M., Gottlieb, G.J., Greider, C., and DePinho, R.A. (1999). Longevity, stress response, and cancer in aging telomerase-deficient mice. Cell 96, 701-712.

Sasa, G., Ribes-Zamora, A., Nelson, N., and Bertuch, A. (2012). Three novel truncating TINF2 mutations causing severe dyskeratosis congenita in early childhood. Clin Genet, 81,470-478.

Schlapbach, C., Yerly, D., Daubner, B., Yawalkar, N., and Hunger, R.E. (2011). Telomerase-specific GV1001 peptide vaccination fails to induce objective tumor response in patients with cutaneous $\mathrm{T}$ cell lymphoma. J Dermatol Sci 62, 75-83.

Schoeftner, S., and Blasco, M.A. (2008). Developmentally regulated transcription of mammalian telomeres by DNA-dependent RNA polymerase II. Nat Cell Biol 10, 228-236.

Seger, Y.R., Garcia-Cao, M., Piccinin, S., Cunsolo, C.L., Doglioni, C., Blasco, M.A., Hannon, G.J., and Maestro, R. (2002). Transformation of normal human cells in the absence of telomerase activation. Cancer Cell 2, 401-413.

Shaw, V.E., Naisbitt, D.J., Costello, E., Greenhalf, W., Park, B.K., Neoptolemos, J.P., and Middleton, G.W. (2010). Current status of GV1001 and other telomerase vaccination strategies in the treatment of cancer. Expert Rev Vaccines 9, 1007-1016.

Shay, J.W., and Wright, W.E. (2004). Telomeres in dyskeratosis congenita. Nat Genet 36, 437-438.

Smogorzewska, A., Karlseder, J., Holtgreve-Grez, H., Jauch, A., and de Lange, T. (2002). DNA ligase IV-dependent NHEJ of deprotected mammalian telomeres in G1 and G2. Curr Biol 12, 1635-1644.

Stansel, R.M., de Lange, T., and Griffith, J.D. (2001). T-loop assembly in vitro involves binding of TRF2 near the 3 ' telomeric overhang. EMBO J 20, 5532-5540.

Touzot, F., Callebaut, I., Soulier, J., Gaillard, L., Azerrad, C., Durandy, A., Fischer, A., de Villartay, J.P., and Revy, P. (2010). Function of Apollo (SNM1B) at telomere highlighted by a splice variant identified in a patient with Hoyeraal-Hreidarsson syndrome. Proc Natl Acad Sci U S A 107,10097-10102.

van Overbeek, M., and de Lange, T. (2006). Apollo, an Artemis-related nuclease, interacts with TRF2 and protects human telomeres in S phase. Curr Biol 16, 1295-1302.

van Steensel, B., Smogorzewska, A., and de Lange, T. (1998). TRF2 protects human telomeres from end-to-end fusions. Cell 92, 401413.

Walne, A.J., Vulliamy, T., Beswick, R., Kirwan, M., and Dokal, I. (2008). TINF2 mutations result in very short telomeres: analysis of a large cohort of patients with dyskeratosis congenita and related bone marrow failure syndromes. Blood 112, 3594-3600.

Wang, F., Podell, E.R., Zaug, A.J., Yang, Y., Baciu, P., Cech, T.R., and Lei, M. (2007). The POT1-TPP1 telomere complex is a telomerase processivity factor. Nature $445,506-510$.

Wang, R.C., Smogorzewska, A., and de Lange, T. (2004). Homologous recombination generates T-loop-sized deletions at human telomeres. Cell 119, 355-368.

Wright, W.E., Piatyszek, M.A., Rainey, W.E., Byrd, W., and Shay, J.W. (1996). Telomerase activity in human germline and embryonic tissues and cells. Dev Genet 18, 173-179.

Wright, W.E., Shay, J.W., and Piatyszek, M.A. (1995). Modifications of a telomeric repeat amplification protocol (TRAP) result in increased reliability, linearity and sensitivity. Nucleic Acids Res 23, 3794-3795. Wu, P., Takai, H., and de Lange, T. (2012). Telomeric 3' overhangs 
derive from resection by Exo1 and Apollo and fill-in by POT1bassociated CST. Cell 150, 39-52.

Wu, P., van Overbeek, M., Rooney, S., and de Lange, T. (2010). Apollo contributes to $\mathrm{G}$ overhang maintenance and protects leading-end telomeres. Mol Cell 39, 606-617.

Xin, H., Liu, D., and Songyang, Z. (2008). The telosome/shelterin complex and its functions. Genome Biol 9, 232.
Ye, J., Lenain, C., Bauwens, S., Rizzo, A., Saint-Leger, A., Poulet, A., Benarroch, D., Magdinier, F., Morere, J., Amiard, S., et al. (2010a). TRF2 and apollo cooperate with topoisomerase 2alpha to protect human telomeres from replicative damage. Cell 142, 230-242.

Ye, J., Wu, Y., and Gilson, E. (2010b). Dynamics of telomeric chromatin at the crossroads of aging and cancer. Essays Biochem 48, 147-164. 